\title{
Actividad poltergeist y el caso "Andrés Venier": Algunas consideraciones neuropsicológicas, fenomenológicas y psicodinámicas
}

\author{
Alejandro Parra
}

En este artículo el autor plantea la fenomenología, neuropsicología y

psicodinámica en torno a la actividad poltergeist, con particular énfasis en el caso del joven Andrés Venier, de Río Tercero, en la provincia de Córdoba, Argentina. El fenómeno parece estar ocasionado por psicokinesis (influencia de la mente sobre la materia) a nivel inconsciente. Estos eventos se vienen reportando desde la Antigüedad. Su fenomenología es descrita en términos de lanzamientos y desplazamientos de objetos, ruidos, luces, apariciones y olores fétidos. Además, los poltergeist son capaces de interferir equipos electrónicos mientras que otros reportes incluyen lesiones somáticas.

Actividad poltergeist - neuropsicología

Poltergeist activity and Andrés Venier's case: some phenomenological, neuropsychological, and psychodynamical issuess

In this article the author discusses the phenomenology, neuropsychological and psychodynamical issues about poltergeist activity, with main emphasis on Andrés Venier's case, of Rio Tercero, province of Córdoba, Argentina. The phenomenon seems to be caused by unconscious psychokinesis (mind over matter). These events have been reported since antiquity. Its phenomenology is described in terms of throwings and displacements of objects, noises, lights, apparitions and foul smellings. Besides, poltergeists are capable of interference with electronic equipment while other reports include somatic lesions.

Poltergeist activity - neuropsychology

Correo electrónico: rapp@fibertel.com.ar 


\section{INTRODUCCIÓN}

Tradicionalmente, el así llamado fenómeno poltergeist ha estado asociado a espíritus inquietos o traviesos, pero que a veces también se comportan agresivamente. La palabra poltergeist proviene del alemán polter, "golpear", y geist, "espíritu". Algunos casos de poltergeist no han sido cabalmente explicados y podrían implicar la presencia de inteligencias desencarnadas. En otros casos, el fenómeno parece ser ocasionado por la psicokinesis (PK) inconsciente de una persona (Cassirer, 2001; Houran \& Lange, 2001; Spencer \& Spencer, 1997; Stander \& Schmolling, 1996).

La actividad poltergeist tiene una fenomenología típica descrita por varios autores de diversas maneras. Los más frecuentes son lluvias de piedras, de polvo y de otros objetos pequeños; lanzamientos y desplazamientos de objetos (incluyendo muebles pesados); ruidos intensos, luces, apariciones y olores fétidos. Los poltergeist son capaces de interferir los teléfonos y otros equipos electrónicos, y de encender y apagar las luces y cualquier aparato eléctrico. Algunos sujetos victimizados por la actividad poltergeist reportan lesiones somáticas, como pellizcos, mordidas, golpes y hasta agresiones sexuales a las personas que los experimentan (Auerbach, 1986; Carrington \& Fodor, 1951).

Asimismo, la actividad poltergeist, por lo general, comienza y termina de manera abrupta. Un episodio típico puede durar desde algunas horas hasta varios meses, e incluso se ha informado de algunos que se prolongaron durante años. La actividad casi siempre ocurre cuando alguien está presente, generalmente en torno a un "agente", aquel que parece servir como foco o imán de la actividad. El agente es un factor en la mayoría de los casos, tanto de los que parecen de etiología espírita como de los que pueden ser provocados por psicokinesis humana (Rogo, 1979, 1986; Roll, 1972). En el transcurso de los siglos, las descripciones de incidentes inexplicables en el mundo físico presentan una uniformidad notable. Por los trabajos de G. N. M. Owen (1964), Thurston (1953), entre otros, se han observado similitudes asombrosas a través de los siglos y en todos los países.

Desde la Antigüedad se viene informando en todo el mundo de la existencia de disturbios provocados por la actividad poltergeist (Bozzano, 1925; Flammarion, 1952; Thurston, 1953). Sin embargo, a fines del siglo XIX, la investigación de los fenómenos psíquicos permitió estudiar más críticamente este tema. Entre los primeros investigadores se contaban los fundadores de la Society for Psychical Research (SPR), sir William Barrett y Frederic W. H. Myers (Auerbach, 1986; Myers, 1903).

En los años treinta del siglo XX, el psicólogo e investigador psíquico Nandor Fodor elaboró la teoría de que algunos disturbios de poltergeist no eran 
causados por espíritus, sino por agentes humanos que sufrían de una intensa ira reprimida, hostilidad y tensión sexual. Fodor tuvo éxito en demostrar su teoría en varios casos, incluyendo el famoso caso Thornton Heath, ocurrido en Inglaterra, que Fodor investigó en 1938. Se trataba de una mujer cuyas represiones provocaban un brote de fenómenos poltergeist $\mathrm{y}$, al parecer, el ataque de un vampiro. Fodor fue duramente criticado por los espiritistas, a los cuales ganó un pleito por calumnias difundidas en uno de sus periódicos.

Las investigaciones llevadas a cabo por W. G. Roll y sus colegas (Roll, 1964, 1968, 1970, 1972, 1993; Roll, Burdick \& Joines, 1973; Roll, Burdick \& Joines, 1974; Roll \& Gearhart, 1974; Roll, Maher \& Brown, 1992; Roll \& Montagno, 1983; Roll \& Pratt, 1971; Roll, Sheehan, Persinger \& Glass, 1996; Roll \& Stump, 1969; Roll \& Tringale, 1983) han intentado probar -al menos en parte- una relación entre la disfunción psicológica y la actividad poltergeist. En los años sesenta Roll comenzó a estudiar 116 casos ocurridos a lo largo de cuatro siglos en más de cien países, y pudo identificar la repetición de lo que el investigador acuñó como "psicokinesis espontánea recurrente" (PKER), que son efectos materiales espontáneos y explicables. También descubrió que el agente más frecuente era un niño o adolescente cuya PK involuntaria constituía una manera de expresar su hostilidad sin temor a ser castigado. La persona generalmente ignoraba que era la causa de los disturbios, pero secreta o abiertamente se alegraba de ellos. Su hipótesis está hoy en día tan divulgada que aparece incluso mencionada en varios libros de texto de psicología y psicoanálisis, así como en numerosos documentales de televisión. En algunos casos, la psicoterapia hace desaparecer los fenómenos poltergeist.

\section{FENOMENOLOGÍA DE LA ACTIVIDAD POLTERGEIST}

De toda la casuística anómala, quizá la actividad poltergeist haya cautivado a numerosas editoriales y emisoras de televisión, las cuales han producido literatura y documentales de variada calidad que, a nivel popular, ha impresionado por estos relatos. El tema se ha prestado a menudo para interpretaciones sensacionalistas a causa del testimonio de las personas victimizadas, su vinculación con leyendas folclóricas locales y las terapias y/o intervenciones para su tratamiento e investigación. El fenómeno está tan ampliamente divulgado y reportado en todas las épocas y culturas que se ha bautizado a estos sitios así afectados como "casas embrujadas", "casas endemoniadas", "hechizadas", "moradas mal-assombradas" (en portugués) o "infestadas" (haunting, en inglés) (Aguilar, 1995; Friderichs, 1980; Guimaraes Andrade, 1988; Jordán Peña, 1982; Machado \& 
Zangari, 1995; Percia de Carvalho, 1992; Tinoco, 1978).

A fines de los años setenta, Alan Gauld y A. D. Cornell (1979) llevaron a cabo un análisis de 500 casos ocurridos en todas partes del mundo desde 1800. Estos investigadores identificaron 63 características generales; el 64 por ciento de los casos había consistido en movimientos de objetos pequeños; el 58 por ciento fueron más intensos por la noche; en el 48 por ciento hubo objetos golpeados; el 36 por ciento consistía de movimiento de objetos de gran tamaño; el 24 por ciento duró más de un año; en el 16 por ciento hubo comunicación entre el poltergeist y el agente; en el 12 por ciento hubo abrir y cerrar de puertas y ventanas. Antes del siglo XIX, las manifestaciones de los poltergeist eran atribuidas al diablo $\mathrm{y}$ otros demonios, así como en brujas y espíritus de los muertos. El análisis de Gauld y Comell mostró que solo un 9 por ciento de los casos eran atribuidos al diablo, el 7 por ciento a brujas y el 2 por ciento a espíritus. La mayoría de los casos atribuidos a demonios ocurrieron en países no occidentales.

El caso ha sido estudiado desde una perspectiva fenomenológica por el oficial de la policía francesa Émile Tizané (1977), cuyo análisis se basa en centenares de casos ocurridos entre 1925 y 1950. Entre las características observadas por Tizané se advierten caídas de piedras que a menudo rompen los cristales de las ventanas o penetran por las aberturas; ruidos en las puertas, las paredes o los muebles; puertas, ventanas e incluso armarios bien cerrados que se abren por sí solos; objetos que son insólitamente cambiados de sitio o arrojados lejos (los objetos más frágiles no se rompen, aun cuando sufran una caída de bastantes metros, mientras que otros, más sólidos, se destrozan por completo), en ocasiones los objetos desplazados no presentan una trayectoria regular, se comportan como si alguien los transportara y a veces siguen los contornos de los muebles. Algunos ejemplos demuestran la existencia de objetos que penetran en un espacio cerrado, tienen cambios de temperatura (más calientes) $\mathrm{u}$ objetos que parecen adoptar una forma en el aire.

Un examen más detallado de la psicología de los individuos que ocuparon lugares centrales en algunos casos de actividad poltergeist la hace aparecer como más rica que sus estudios físicos. Roll (1972) sostiene que en los casos reportados antes de 1900, cerca del 80 por ciento de las personas epicentro eran mujeres, pero en este siglo la distribución por sexos ha cambiado notablemente, y ambos sexos están igualmente afectados por la actividad poltergeist. Roll estableció también que la edad promedio de esas personas era de 16 años.

En un reciente estudio llevado a cabo por nosotros (Gómez Montanelli \& Parra, 2002) acerca de 13 experiencias psi y relacionadas con psi, encontra- 
mos que el 50,6 por ciento $(\mathrm{N} 1=432)$ de creyentes en lo paranormal respecto a 23,2 por ciento de estudiantes de psicología $(\mathrm{N} 2=392)$ respondieron afirmativamente a la pregunta (P1): ¿Ha observado usted anormalidades en el funcionamiento de aparatos eléctricos, computadoras $u$ otro equipo que le hayan parecido muy extrañas, y que, hasta donde usted ha podido determinar, no fueron debido a causas normales $o$ naturales? Otra pregunta (P2): ¿Le ha sucedido a usted escuchar golpes y/o encontrar vidrios rotos o astillados $y / u$ objetos quemados, sin que hubiera una causa fisica aparente?, fue respondida en el 40 por ciento-22,7 por ciento, respectivamente. Tomando como ejemplo la muestra de estudiantes (N2), encontramos que al menos una vez en su vida el 54,9 por ciento $(\mathrm{P} 1 \mathrm{~N} 2=91)$ y el 32,5 por ciento $(\mathrm{P} 2 \mathrm{~N} 2=89)$ respondió haber tenido la experiencia PKER, en tanto que el 32,5 por ciento y el 67,4 por ciento varias veces. Esto indicaría que las experiencias PKER son más frecuentes de lo que se cree, aunque probablemente menos reportadas o menos identificadas como disfunciones del entorno causada por "psi".

No menos interesante es el grado de perturbación psicológica (conflictividad) para tales experiencias. Siguiendo el mismo ejemplo, bajo la pregunta: ¿Han resultado estas experiencias de algún modo traumáticas o conflictivas?, encontramos que el 60,4 por ciento $(\mathrm{P} 1 \mathrm{~N} 2=91)$ y el 65,1 por cien- to $(\mathrm{P} 2 \mathrm{~N} 2=89)$ había resultado perturbadora. Poco más de la mitad, para ambas preguntas, respondió que la PKER había resultado, en términos de intensidad, menos perturbadora respecto de un número mucho menor de casos (aproximadamente cuatro), quienes la habían considerado intensamente perturbadora.

Sin embargo, cuando comparamos ambas muestras (N1 y N2), encontramos una diferencia significativa para las dos preguntas respecto de la perturbación emocional de la experiencia de PKER y su intensidad $(\mathrm{p}<.0001)$ que indica que la muestra de estudiantes reporta menos experiencias pero mayor perturbación emocional respecto de la de creyentes, quienes reportan más experiencias pero menos perturbación. Estos resultados muestran claramente que las experiencias de PKER tienden a ser más reportadas por los creyentes en lo paranormal, quizá no solo debido -probablemente- a sus convicciones espiritualistas respecto de las de los estudiantes $(\mathrm{N} 1=432$ vs. $\mathrm{N} 2=392, U$ de Mann Whitney $p<.0001$, a una cola), sino también a un mayor grado de significación conceptual de la experiencia PKER en los creyentes que en los estudiantes, quienes probablemente sienten más perturbación emocional por su falta de significación conceptual. Además, es notable que esta tendencia se repite en otras experiencias psi y relacionadas con psi. 


\section{ACTIVIDAD POLTERGEIST: UNA INTERPRETACIÓN PSICODINÁMICA}

No obstante la noción tradicional de que la tensión sexual y las frustraciones son las causas principales que dan lugar a las manifestaciones poltergeist, esta idea ha sido plenamente explotada por la cinematografía en filmes como El exorcista, El Ente y Carrie. En cierto modo, era natural que los primeros investigadores consideren que la pubertad, la tensión sexual y el fenómeno poltergeist estén de algún modo relacionados. Muchos casos de poltergeist se centran en torno a jóvenes púberes y, en ocasiones, los efectos incluyen ataques de claro significado sexual. De hecho, posteriormente, los casos de poltergeist han venido extendiéndose hasta afectar casi en igual número a ambos sexos, circunstancia que disminuye la posibilidad de que guarden relación con la sexualidad femenina frustrada.

En algunos casos, los niños y las niñas afectados por el fenómeno que declararon que sufrían de algún mal (o varios) de naturaleza psíquica: histeria, depresión, agresividad (en exceso o por defecto, en último caso conducente a la represión) o neurosis fueron examinados por psiquiatras o psicoanalistas. Un problema importante en relación con esos diagnósticos es que el psiquiatra que reconoce al niño está predispuesto al hallazgo de anormalidades funcionales. Por el contrario, si el psiquiatra ig- nora la razón por la que se lleva al niño a la consulta, es posible que diagnostique neurosis, si bien es posible que el niño se haya vuelto neurótico a causa de la persecución del poltergeist. El único modo posible de diagnosticar sería disponer de un perfil de la personalidad del niño, trazado antes del comienzo de la actividad poltergeist. Existe una prueba más firme que sugiere que la actividad poltergeist no está relacionada con la frustración sexual o la enfermedad mental, sino con el deseo de llamar la atención. En un estudio de jóvenes de ambos sexos, menores de 18 años, entre los cuales algunos habían sido foco de actividad poltergeist, Roll encontró que no menos del 62 por ciento estaba viviendo fuera de su hogar cuando comenzó. De los demás, un 17 por ciento, solo vivía en el hogar o se hallaba presente en él uno de sus padres. Aunque estas cifras no son elevadas, no podemos establecer la comparación con los niños normales. Caben pocas dudas de que la carencia de ambiente familiar o un hogar inestable tiende a reforzar la idea de que los poltergeist puedan ser "instrumentos" utilizados por el sujeto para llamar la atención.

Sigmund Freud fue el primero en tomar contacto con la actividad poltergeist por influencia de su colega Carl G. Jung. Eva Brabant (1994) sostiene que Freud y Ferenczi, un neurólogo húngaro que contribuyó a la construcción de la teoría psicoanalítica, mantu- 
vieron correspondencia respecto de eventos de tipo poltergeist (en esta colección son 483 de un total de 1.236 cartas escritas durante 25 años). En una carta fechada en 1909, Ferenczi escribía sobre la visita a una médium, "Frau Seidler", que, él creía, le había leído los pensamientos a Freud. El psiquiatra le respondió: "Sólo ahora me recuperé del shock y no puedo dejar de confrontar con el asunto como cualquier otro (...) Entretanto, mantengamos silencio sobre todo esto".

El psicoanalista Nandor Fodor, en su obra Freud, Jung and occultism (Fodor, 1971) menciona el interés de Jung por los aspectos psicológicos de los fenómenos ocultos. En esta fase preliminar de su trabajo, Jung concluyó que “... el desarrollo mediúmnico representa un intento de desarrollar el carácter a través de una nueva forma...". Fodor reveló que en una de sus conferencias, "Los fundamentos psicológicos de la creencia en espíritus", el 4 de julio de 1919, Jung aplicó específicamente su teoría al fenómeno poltergeist desencadenado en el transcurso de la sesión espírita por los médium. Sostenía, en esa ocasión, que "los espíritus eran complejos inconscientes y autónomos que aparecen como proyección". A la pregunta sobre quién o qué proyecta esa energía a fin de producir la actividad poltergeist, Jung respondió que el origen estaba en un médium vivo, el cual sería la "exteriorización de complejos inconscientes". Sin embargo, después de una sesión espírita de 1925, en la casa del doctor Rudolph Bemouilly, de Zurich, el investigador dudó de que el fenómeno pudiese ser explicado como producto de la energía PK de un ser vivo. Jung fue testigo de una variedad de fenómenos de tipo PK, así como la materialización de miembros humanos. Después de la sesión ya no se mostraba tan seguro de sus primeras conclusiones y de que "una aproximación exclusivamente psicológica no podía hacer justicia al fenómeno en cuestión". Jung extendió sus opiniones acerca del fenómeno poltergeist, sosteniendo la creencia de que todos poseen potencial para lograr efectos PK a partir de su propia energía.

Es preciso realizar más investigaciones y profundizar los estudios en este campo antes de extraer conclusiones definitivas. Si se busca una explicación psicológica de la razón de los efectos poltergeist, posiblemente deberíamos acercarnos al problema empezando por observar a qué conduce la necesidad de atención en un medio carente de afectos. En el caso de los adolescentes, la necesidad de atención puede estar reforzada por ser una etapa de crisis e incertidumbres.

De todos modos, resulta obvio que esta especulación no puede brindar una explicación completa. Muchos niños buscan llamar la atención, pero pocos de ellos generan a su alrededor actividad poltergeist. 
MODERNAS INTERPRETACIONES DE LA ACTIVIDAD POLTERGEIST

En años recientes se vienen haciendo estudios muy rigurosos de la actividad poltergeist, sobre todo considerando el reporte de los testimonios de estos eventos. Johan Gerding y Rens Wezelman, del Parapsychology Institute de Amsterdam, junto con Dick Bierman, de la Universidad de Utrecht (Gerding, Wezelman \& Bierman, 1997), reportaron un caso de perturbación poltergeist en una familia turca de Druten, en Holanda, en la primavera de 1995. Vuelo de piedras y otros objetos acabaron por convertir al hijo de la familia, de 15 años, en el epicentro de actividad poltergeist. Mediante el análisis de los testimonios de sus vecinos, amigos y oficiales de policía, quienes testificaron la autenticidad de los fenómenos, los investigadores efectuaron algunos estudios experimentales con un juego similar al RNG (Generador de Eventos Aleatorios) para probar influencias PK y se instaló un equipo de RNG para observar fluctuaciones en la coherencia del ordenamiento de la secuencia aleatoria. El estudio determinó una breve pero intensa relación entre las fluctuaciones anómalas del RNG durante los periodos en que se relataban las perturbaciones.

Andrew Nichols y William Roll (1999) estudiaron los aspectos electromagnéticos y neuropsicológicos del caso del llamado "poltergeist de agua" de
Jacksonville, que ocurrió en una residencia en la Florida entre noviembre de 1996 y enero de 1997, y su característica más destacable, además de los movimientos anómalos de objetos, es la inexplicable aparición de grandes cantidades de agua. Fort, Thurston (1953), Gauld \& Cornell (1979), Bender, Bayless y Rogo $(1979,1986)$ citan numerosos casos donde apariciones de agua acompañan los efectos espontáneos de PK típicos de los poltergeist. Los test psicológicos proyectivos revelaron que uno de los testimonios se adecuaba al perfil de la "personalidad poltergeist": típicamente un adolescente, con baja tolerancia a la frustración, reprimiendo sentimientos de agresión y hostilidad. El cuestionario neuropsicológico sugirió que dos testimonios, entre ellos el aparente agente, podrían tener labilidad en el lóbulo temporal. En relación con las mediciones físicas, Nichols y Roll verificaron que la fuerza de los campos magnéticos de los locales donde los fenómenos habían ocurrido era significativamente diferente de otros sitios.

De acuerdo con el procedimiento de evaluación cuantitativa, el físico William Everist llevó a cabo dos estudios de sensibilidad aparicional con la cooperación de participantes sensitivos y no-sensitivos, estos últimos estudiantes de un curso de parapsicología, quienes debían tratar de determinar qué lugares de la casa eran los "encantados". El estudio se desarrolló en una antigua casa en Bisbee, Arizona, en la que su dueño 
señaló en un mapa los lugares donde se habían observado las apariciones fantasmales. Doce estudiantes fueron pareados en seis grupos, compuesto uno por un sensitivo y otro un no-sensitivo, y se les entregó un mapa de la casa a cada par de participantes. En forma independiente, debían determinar los lugares o habitaciones de la casa donde intuían o pensaban que estaba "encantado", en comparación con otros sitios de control (no-encantados). Tanto los sensitivos como los estudiantes desconocían en qué sitios habían ocurrido los eventos poltergeist (en este caso experiencias aparicionales o "sitios visitados por fantasmas"). Los sujetos visitaban cada lugar de la casa señalizado por el investigador, de acuerdo con las declaraciones del dueño, pero esta marca no indicaba si el lugar había sido visitado o no por el fantasma. Se llevaron a cabo 20 visitas a la casa, diez señaladas como "encantadas" y otras diez de control. Cuando se recogieron los datos, los participantes obtuvieron un promedio significativamente más alto de aciertos, señalando los lugares "encantados" en comparación con los lugares de control, y de este grupo, los sensitivos obtuvieron un promedio más alto de aciertos con respecto al grupo de no-sensitivos (estudiantes) en determinar qué sitios habían sido "visitados." Los aciertos se determinaban indicando sensaciones emocionales, físicas, cenestésicas o cualesquiera otras, toda vez que el participante, sea sensitivo o no, visitaba cada habitación completando un cuestionario, apoyando la creencia que parece confirmar lo que muchas personas reportan como experiencias de contacto espiritual.

William Roll y Andrew Nichols advirtieron un aumento en el campo geomagnético y un elevado número de anomalías fotográficas, como la aparición de burbujas traslúcidas del tamaño de globos esféricos que aparecían en fotografias digitales, que coincidía significativamente con un aumento en la actividad geomagnética y una alta concentración de iones en la atmósfera. Nichols y Roll (2000) reportaron los eventos en la casa McRaven en Vicksburg, Mississippi, y la casa Aponawicz, en Pennsylvania, y logró filmar estas apariciones esféricas, que aparecen espontáneamente, moviéndose en trayectoria curva. Roll sostiene que los testigos de eventos poltergeist en antiguos castillos (por ejemplo en Dragsholm y en Engso), incluían manifestaciones de PK y apariciones, principalmente de personajes legendarios, el sonido de cadenas y pasos, o apariciones luminosas, captadas mediante sensibles cámaras infrarrojas y detectores Geiger. Estos estudios consisten en introducir artefactos de medición sensibles para detectar cambios anómalos del campo electromagnético e impresiones de sujetos, quienes decían "percibir" dichas anomalías. Los investigadores compararon los relatos de los psíquicos y correlacionaron tales descripciones con 
los registros de anomalías geofísicas y hallaron resultados interesantes, principalmente en relación con la descripción de los psíquicos, quienes fueron localizados al azar en diferentes sitios de ambos castillos, desconociendo el lugar donde los testigos afirmaban "sentir" la presencia de apariciones. Algunos de los sitios mencionados por los psíquicos coincidieron con la descripción de los testigos y esta información se correlacionó con las detecciones de los equipos electrónicos (Roll \& Persinger, 1998).

En base a estos trabajos, y en una investigación reciente, Roll y Persinger (1998) sugieren que la RSPK funcionaría mediante la energía electromagnética (EM) del ambiente, la cual sería modulada por la EM del agente y direccionada sobre objetos significantes para el sujeto, incluyendo también estudios neurológicos $\mathrm{y}$ psicodinámicos llevados a cabo con los agentes PKER, los cuales mostraron tensiones psicosociales y epilepsia. De este contexto surgiría un "perfil EM" capaz de modular y direccionar la EM del ambiente. La PKER podría actuar como un fenómeno físico con un aspecto humano, de acuerdo con la teoría del "campo psi" de Roll.

Más recientemente, Richard Wiseman, Caroline Watt y Emma Greening, Paul Stevens y Ciaran O'Keefe (1997) unieron esfuerzos para investigar las variables psicológicas y los campos magnéticos del palacio de Hampston, alrededor del cual muchas personas vienen reportando fenómenos inusuales en muchos sitios de este. Estos investigadores encuestaron a 600 personas acerca de su creencia en fantasmas y otros fenómenos inusuales. Quienes creían en fantasmas reportaron más eventos anómalos que los que no creían, o eran significativamente más propensos a atribuir los fenómenos a entidades desencarnadas. Antes de visitar los sitios, a la mitad de los participantes se les dijo que el área estaba asociada con un aumento de fenómenos inusuales, mientras que a los otros se les dijo lo opuesto. En línea con estudios sobre la psicología de la creencia en lo paranormal, hubo un mayor número de experiencias inusuales reportadas por aquellos que creían en fantasmas que quienes no creían.

William T. Joines y William Roll propusieron dos teorías físicas para la psicokinesis espontánea recurrente (PKER). La primera está basada en uno de los principales problemas para el investigador de PKER: identificar la energía que causa los movimientos de objetos. Tal energía afecta los objetos que están asociados con los individuos con quienes el agente tiene relación. Para comprender la PKER, proponen que el agente enlaza las fluctuaciones aleatorias de la energía de punto-cero (EPC), un plenum de energía electromagnética que abarca el espacio e interactúa con la gravitación y la inercia. El agente no generaría la energía para la PKER, sino 
que manipularía las fluctuaciones aleatorias del vacío de modo de reducir la inercia y la gravedad que normalmente mantiene un objeto en su lugar. Si la PKER utiliza la EPC esto demuestra que el vacío tiene un componente consciente. Joines ya había sugerido que el proceso PKER tiene relación con ondas psi del agente, que resultan en efectos focales y atenuación de los incidentes de PKER con la distancia del agente. Si las ondas psi producen una señal coherente dirigida al objeto físico, este proceso quedaría atenuado por las fluctuaciones aleatorias de la EPC que rodean tanto al agente como al objeto. La EPC al mismo tiempo proporcionaría la energía para la PKER que resulta en la reducción de los movimientos de los objetos con la distancia. Joines analizó los efectos de declinación en los casos de Miami, Oliver Hills y Tina Resch desde la teoría del EPC. Los resultados sugieren que el EPC podría ser la conexión entre las ondas psi y las ondas electromagnéticas. Según estos investigadores, el componente energético-emocional de los objetos puede mostrar cuándo los objetos cargados emocionalmente quedan afectados por la PKER (Joines \& RolI, 2001).

\section{El Caso de ANDrés Venier}

La familia está compuesta por el matrimonio de Óscar, de 50 años, y Mónica, de 44. Ninguno de ellos manifiesta haber tenido experiencias paranormales previas, tienen buen nivel cultural (Óscar es industrial y Mónica es profesora de música en el conservatorio de arte) y no están involucrados en temas esotéricos, creencias o supersticiones. Conviven con sus cuatro hijos, quienes concurren a la escuela secundaria.

Es una familia absolutamente normal, excepto por una sola cosa. La casa de la calle Guillermo Marconi 1821 casi no tiene un solo vidrio sano, y algunos testimonios sorprenden. Toda la familia, incluso el comisario - que se hizo presente en la casa-, familiares y vecinos, lo atribuyen a cierto "poder" de Andrés, de 18 años. Un total de 16 policías, apostados en torno a la casa, a plena luz, no pudieron identificar quién arrojaba las piedras, algunas de las cuales efectuaban trayectorias imposibles. Impactan en los vidrios con una puntería asombrosa. Si la causa del fenómeno tuviera lugar por motivos fraudulentos (Andrés $u$ otro en complicidad arroja las piedras con la fuerza de su mano), esto respondería al efecto de tirador asombrosamente hábil para engañar, durante los últimos cuatro meses, a los cinco miembros de su familia $\mathrm{y}$ vecinos en donde cualquier manipulación sería fácil de detectar.

Por lo general, los fenómenos tienen lugar en la casa, nunca fuera de ella. Quizá el dato característico de este caso es la rotura de vidrios de ventanas, de tres a cinco agujeros de hasta 12 centímetros de diámetro en las persianas de plástico, otros en cortinas de 
tela, y, más recientemente, un espejo. Todo comenzó la noche del 25 de febrero con una piedra que rompió un vidrio. La noche del 26, su madre, Mónica Hermandorena, una de las principales testigos, indicó que el suceso de piedras "fue una especie de bombardeo" que se extendió por seis horas, al otro día el suceso duró aproximadamente diez horas, y luego continuaron por intervalos irregulares, pero con más intensidad. El tamaño de las piedras -típicas de la zona de las sierras cordobesas, que se encuentran en el camino hacia la casa Venier- era más grande con el transcurrir de los días, algunas de ellas del tamaño de un huevo de gallina. Su peso alcanzaba los 1,3 kilogramos. La actividad paranormal -caracterizada específicamente por el desplazamiento de piedras a gran velocidad y con gran violencia- tuvo dos periodos: uno entre el 26 y el 29 de febrero, y el segundo entre el 1 y el 3 de marzo. Los eventos luego continuaron durante varios periodos, entre el 16-18 y 25-28 de abril, 2 y 3, 12-18 de mayo y continuaron por periodos más cortos (un día o dos), incluso al momento de escribir el presente artículo. Uno de los “desplazamientos", quizá el más singular, ocurrió en el baño de la casa, uno de los lugares donde -en el momento de mi visita- fui sorprendido por la aparición de una de las piedras. Alertados por esto, la familia y yo corrimos hacia allí y descubrimos que la piedra había aparecido inexplicablemente en ese lugar.

En su intento de buscar una explicación racional a estos fenómenos, Mónica sostiene que al principio ella y su familia pensaron que alguien lanzaba las piedras, hasta que la policía y el comisario local, después de tres días de observación, análisis e hipótesis, descartaron esta hipótesis a causa de sus raros "trayectos" con que se desplazaban. Además de la falta de un "autor" de estos eventos -cualquier persona debería ser razonablemente visible- el comisario inspector zonal, que intervino más de una vez alertado por las denuncias de los "ataques" a la familia, fue incapaz de explicar el trayecto de una de las piedras, la cual, con inusitada violencia, destrozó el vidrio de una de las ventanas de la casa ante el oficial de policía. A causa de ello, algunos vecinos alertaron al cura local temiendo por la presencia de 'espíritus'. Pero tres sacerdotes católicos que visitaron la casa dijeron que no había absolutamente nada. Así lo manifestaron dos psíquicos, de manera independiente, que también la visitaron, Daniel Enrici y Marcelo Berdini, quienes indicaron haber sentido una "poderosa fuerza energética". Ni Mónica ni su familia atribuyen este fenómeno a espíritus de los muertos, duendes ni otra entidad sobrenatural.

Sorprendentemente, ninguna piedra golpeó jamás a ningún miembro de la familia. Mónica relata el comportamiento anómalo de una de las piedras: 
Estaba con mi hijo Ezequiel, preparándole para un examen, sentados frente a la ventana del frente de la casa, cuando una piedra pasó entre los dos sin golpearnos y se detuvo sobre la mesa, frente a nosotros, cuando debería haber resbalado en la superficie de la mesa o haber ido más lejos, teniendo en cuenta la velocidad con que la piedra ingresó (Parra, 2004).

La actividad poltergeist, por lo general, comienza y termina de manera abrupta. Un episodio típico puede durar horas o varios meses, e incluso algunos se han prolongado durante varios años. En este caso, los fenómenos se tornan más frecuentes e intensos cuando su hijo Andrés está despierto. En ocasiones, cuando él está en la casa, han entrado piedras, casi siempre en su presencia. De lo contrario no ocurre nada. Esto sugiere que Andrés sería el agente o "epicentro" de actividad psicokinética (PK); aquel que parece servir como foco o imán. En general, las piedras se desplazan violentamente hacia ventanas y destruyen vidrios. De manera sorprendente, de un número de 20 ataques con piedras a vidrios y al aparato de televisión en solo dos ocasiones las piedras "equivocaron" su rumbo e impactaron sobre un mueble o la pared.

En este sentido, el brote de actividad PK (presumiblemente a causa de Andrés) ocurrió durante periodos de mayor intensidad. El primero de estos brotes ocurrió durante seis días continuos, luego cuatro, y más tarde solo dos roturas por semana. Durante un largo periodo (17 días) no hubo rotura alguna ni se observó otro fenómeno, sin embargo coincidió con el periodo en el que Andrés recibió una importante dosis de medicamentos e incluso internación por descompensación medicamentosa. Es notable observar los agujeros producidos por el ingreso de las piedras (desde el patio hacia dentro de la casa). En un periodo no mayor de un mes, una literal "lluvia de piedras" destruyeron persianas, vidrios de puertas y ventanas, el vidrio del auto de la familia, el vidrio del garaje y la ventana del living-comedor.

Mónica manifiesta preocupación y desconcierto. El martes 1 de junio, Andrés había comenzado una nueva medicación, a causa de la cual permaneció dormido durante la tarde. Mónica relata:

Durmió hasta las 15:30 horas, se levantó y almorzó en la cocina. Yo, por supuesto, ya había tomado la precaución de cerrar todos los postigones y puertas. No había lugar posible por donde ingresara piedra alguna. Sin embargo, estando Andrés parado al lado del refrigerador, junto a mi esposo, sentimos un ruido muy fuerte. Cuando fuimos a ver, la pantalla del televisor estaba rota por una piedra, que quedó incrustada en el aparato de televisión.

Mónica, de inmediato, revisó todas las ventanas y puertas por donde la piedra pudo haber ingresado. "Sin embargo, me topé con que nada podía explicarme cómo aquella piedra pudo haber ingresado a la cocina", manifiesta sor- 
prendida. "Salimos de la casa, acompañadas por Andrés. Cuando regresamos, misteriosamente, otra piedra, que no podemos explicar por dónde ingresó, rompió la cortina y un ventanal". Mónica conservó la pieza de cortina dañada. Aparece con un pequeño agujero, una huella del daño producido por el impacto de la piedra.

Óscar, por su parte, recuerda un caso aún más alarmante: “Andrés y su perra estaban jugando en la cocina, cuando repentinamente sentimos otro impacto sobre el televisor. La pantalla del aparato había quedado enteramente destrozada". Denise, la hija menor del matrimonio Venier, manifestó haber sentido una rara sensación, como una brisa y una vívida sensación de 'presión' sobre sus oídos, justo antes de oír el impacto (ella estaba frente a Andrés), y luego otra piedra impactó fuertemente rompiendo la cortina, el vidrio y el postigón de madera. La noche anterior, Verónica, su hermana mayor, fue testigo de un incidente. Una piedra provino por detrás de ella (Andrés estaba visible), dejó un pequeño raspón en el mueble y el televisor, antes de impactar violentamente en una ventana: "Como si la piedra hubiera girado en una trayectoria inexplicable, para impactar directamente en el vidrio", sostiene Verónica. Y concluye: "No lo puedo explicar".

Al día siguiente, su padre llevó a Andrés en su auto a Villa del Dique, una bonita zona al oeste de la ciudad, para intentar disminuir la tensión fami- liar que les produjo. Mónica nos dijo que aunque Andrés continuó ansioso los fenómenos en la casa habían dejado de ocurrir y, al menos por un periodo, todo volvió a la normalidad. A su regreso, 20 minutos después, otra piedra les dio la bienvenida.

En una ocasión, en presencia del parapsicólogo de la zona, ocurrió un episodio: una piedra ingresó con violencia desde fuera de la casa hasta impactar en un vidrio. Andrés estaba al lado de Miguel. En otra oportunidad, en presencia de Marcelo, ocurrió un episodio en el baño. Una piedra produjo el desprendimiento del protector de policarbonato del cuarto de baño. "Una noche -relata Mónica- Andrés decía que 'alguien' golpeaba la persiana de su cuarto. Nosotros escuchamos los golpes. Aunque la persiana estaba cubierta y bien presionada por un colchón, era imposible que Andrés fuera quien golpeara. Mónica y su hija Verónica escucharon un impacto. "Mi esposo revisó la casa y el patio - continúa diciendo su madre- pero no encontramos nada extraño. Curiosamente, en otras oportunidades escuchamos impactos, hemos visto marcas, pero no encontramos piedra alguna".

Mónica y Óscar han acudido en busca de ayuda a sacerdotes y videntes, han efectuado consultas psiquiátricas y psicológicas. Hubo quienes se ofrecieron a 'exorcizar' la casa, incluso asesorados por el padre Pugnata, de Reducción de la ciudad de Río Cuarto, pero no encontraron solución alguna hasta el presente. 
Mónica tuvo que poner a resguardo el ordenador por temor a que este también termine bajo el impacto de alguna piedra. Ella teme que la computadora sufra daños similares a los ocurridos en los ventanales. "Lo más increíble -sostiene Mónica- es que no dejo de pensar de dónde salen o entran las piedras, ya que no logramos verlas. La casa estaba toda cerrada y dentro de la casa no hay piedras". De hecho, hasta el momento de escribir este artículo, Mónica y Óscar -quienes agradecieron mi presencia en su casa y por "hacer posible que otras personas nos escuchen... que nos sintiéramos más tranquilos y contenidos... y que esta situación tan estresante termine o mejore"- mantienen un permanente registro de los eventos que aún no han cesado. Ambos continúan reportando a mi correo electrónico toda anomalía de la que son testigos. El pasado domingo 20 de junio, Mónica escribió que una piedra rompió el espejo del baño, otra rompió un vidrio de la puerta de la cocina (las puertas de la casa estaban cerradas). Óscar agrega que pocos días después de mi visita, Andrés comenzó nuevamente a comportarse inapropiadamente, se mostraba muy alterado.

Aunque trataron de seguir mis consejos y los del psiquiatra, Andrés continuaba molestando e insultándolos con actitudes hostiles y agresivas. Esta situación produjo un gran estrés. Luego Andrés se calmó y encontró algunas actividades para distraerse.
El informe psicológico de Andrés indica que el joven manifiesta agresiones verbales hacia familiares y extraños, conductas impulsivas y antisociales, lo que dificulta su convivencia familiar y social. Andrés fue sometido también a una serie de exámenes neuropsicológicos, incluidos el test WSC, Trail-Making Test, y la prueba del efecto Stroop. En mi propia visita, yo mismo administré los test proyectivos de dibujos HTP y Familia kinética, que indicaron una incipiente inestabilidad emocional, irritabilidad, impulsividad, sentimientos de inadecuación a su entorno e inmadurez emocional. Su padre refiere que no consigue respetar límites, no respeta a personas mayores y no acepta que lo atiendan, contengan $\mathrm{u}$ orienten. Desde niño Andrés era hiperkinético y presentaba algunas dificultades escolares, al punto que fue diagnosticado con trastorno de déficit atencional. El examen neuropsicológico concluye que Andrés tiene todos los indicadores de un trastorno neurológico, posiblemente un severo déficit del funcionamiento del lóbulo frontal. En efecto, un diagnóstico neurológico indica que Andrés padece una epilepsia refractaria fotosensible y tiene convulsiones desde los 12 años, los principales síntomas de Andrés son lapsus o ausencias irregulares desde los nueve años, en los que pierde levemente la consciencia, pero actualmente se encuentra medicado con antiepilépticos y anticonvulsivantes. A causa de ello, 
Andrés ha venido recibiendo tratamientos psicomotrices, psicopedagógicos, psicológicos, neurológicos y psiquiátricos. Una de las psicopedagogas que evaluó su caso consideró que Andrés podía asistir normalmente a la escuela (con ciertos ajustes curriculares). Su madre también agrega que el joven sufrió muchas restricciones respecto a tareas de recreación, como andar en bicicleta, salir de noche, nadar, manejar una moto o el auto y otras actividades, que ahora debe hacerlo acompañado.

\section{CONCLUSIONES}

Según el psicólogo australiano Harvey Irwin (2004), cerca del 70 por ciento de los casos de "epicentro" de actividad poltergeist son jóvenes menores de 20 años. En los años treinta, el psicoanalista Nandor Fodor elaboró la teoría de que algunos disturbios de tipo poltergeist no eran causados por espíritus, sino por agentes humanos que padecían intensa ira reprimida, hostilidad o represión sexual. Fodor presentó el famoso caso, ocurrido en Inglaterra en 1938, de una mujer cuyas represiones provocaban un brote de actividad PK, en Thornton Heath.

Roll, autor del libro Unleashed: Of poltergeist and murder. The curious story of Tina Resch (Paraview Publishers, 2004), basado en el famoso caso de la actividad PK de la adolescente Tina Resch, en 1984, ha propuesto que la PKER es generada por la energía electromagnética del agente que convierte la energía kinética de los objetos. También ha especulado que estos procesos pueden involucrar la suspensión del campo gravitacional en torno al agente. "Recién ahora estamos comprendiendo el medio electromagnético que llena el espacio e interactúa con la gravedad y la inercia", expresa Roll en un artículo publicado en un reciente número monográfico de la Revista Argentina de Psicología Paranormal, dedicado al fenómeno poltergeist. Roll basa su especulación teórica en torno a la PK espontánea en el modelo del físico Harold Puthoff, quien sostiene que el espacio vacío no está en realidad "vacío" sino que se llena con energía de punto cero (EPC) que permanece activa en cero absoluto cuando todos los efectos térmicos se congelan. Según Roll, Puthoff está desarrollando una tecnología que permita usar la EPC como una fuente de energía renovable y no contaminante para los viajes espaciales. "Para nuestro propósito - sostiene Roll, quien ha mantenido una correspondencia privada con Puthoff- su propuesta más interesante es que la EPC podría ser un mecanismo para la PK espontánea a través de la interacción de la EPC con la gravitación y la inercia".

Para Roll la inercia, esto es, la resistencia de un objeto a la aceleración, es un fenómeno conocido pero que carece de explicación científica. Puthoff considera que la inercia es producto de la 
EPC, y nos proporciona un ejemplo de inercia: "Suponga que usted está parado dentro de un tren. Si el tren deja la plataforma de un tirón, usted puede caerse al piso. ¿Cuál es la fuerza que no parece venir de ninguna parte, que lo golpea para hacerlo caer?".

Puthoff explica que el fenómeno resulta de la aceleración relativa de las estrellas fijas. En el ejemplo del tren, el puñetazo fue dado por la presión de la EPC que llena el espacio entre nosotros y las estrellas. La masa de inercia de un objeto en la tierra resultaría de la masa y la posición de toda la materia en el Universo. Se lo conoce como "El principio de Mach", propuesto por el filósofo austríaco Ernest Mach.

Esta teoría surge de la física quántica, que sostiene que el vacío es un lugar activo. "Si las intenciones conscientes o inconscientes de una persona, como las de un agente poltergeist, pueden interactuar con la EPC, esto significa que el vacío tiene un componente inconsciente", confiesa Puthoff. Nos preguntamos: ¿cuál puede ser la relación entre la EPC y los efectos PKER presumiblemente producidos por Andrés?

Primero, el principal problema para los investigadores de la psicokinesis espontánea ha sido identificar la energía que provoca el movimiento de los objetos. Esta energía afecta a los objetos que están asociados con aquellos individuos con quienes el agente tiene una tensa relación interpersonal. Se- gundo, quizá Andrés quiere comunicar un mensaje o llamar la atención, como un alerta frente a un entorno al cual no consigue adaptarse. Esta desadaptación se proyecta de manera similar al mecanismo psicológico de "desplazamiento de la agresión", pero no a nivel psicológico, sino parapsicológico. Esta ruptura de la hostilidad reprimida toma entonces la forma de energía psicokinética, que obra sobre las frustraciones de Andrés sin que él se dé cuenta de ello, en forma no consciente.

El brote de psicokinesis alivia las intensas frustraciones y tensiones que, indudablemente, podrían causar daño a las personas involucradas, a menos que estas tensiones se puedan elaborar psicológicamente de algún otro modo. Los fenómenos poltergeist bien podrían ser, en parte, "terapéuticos", lo cual explica por qué tales brotes tienen corta vida.

Como ha ocurrido en otros casos, los brotes de poltergeist se comprenden mejor en analogía con los mecanismos de proyección en psicología.

Entonces, los sentimientos de hostilidad reprimidos hacia los padres, figuras vinculadas emocionalmente, también representan el caldo de cultivo para una psicopatología. En el caso de Andrés es evidente que la actividad poltergeist constituye tanto un problema psicopatológico e interpersonal como parapsicológico. 


\section{REFERENCIAS}

Aguilar, F. R. (1995). Las casas que se incendian solas: Psicopirosis en Panamá. Panamá: Universitaria.

Alvarado, C. S. \& Zingrone, N. (1995). Characteristics of haunting with and without apparitions: An analysis of published cases. Journal of the Society for Psychical Research, 60, 385-397.

Auerbach, L. (1986). ESP, hauntings and poltergeists: A parapsychologist's handbook. Nueva York: Warner Books.

Bozzanao, E. (1925). Los fenómenos de encantamiento. Barcelona: Bauza.

Brabant, E. (1994). The correspondence of Sigmund Freud and Sandor Ferenczi. Vol. 1, 1908-1914. Cambridge, MA: Harvard University Press.

Carrington, H. \& Fodor, N. (1951). Haunted people: Story of the poltergeist down the centuries. Nueva York: Dutton.

Cassirer, M. (2001). The hidden powers of nature: Hauntings, poltergeist, mediums seances. Pulborough: D. J. Ellis Publishers.

Flammarion, C. (1952). Las casas de duendes. Buenos Aires: Schapire.

Fodor, N. (1971). Freud, Jung and occultism. Nueva York: University Books.

Friderichs, E. (1980). Casas mal-assombradas: Fenomenos de telergia. Sao Paulo: Loyola.

Gauld, A. \& Cornell, A. D. (1979). Poltergeist. Londres: Routledge \& Kegan Paul.

Gerding, H.; Wezelman, R. \& Bierman, D. (1997). The Druten disturbances: Ex- ploratory RSPK research. En: Wiseman, R. (Ed.). Proceedings of the Parapsychological Association. 4th Annual Convention. Brighton, UK, 97-107.

Gómez Montanelli, D. \& Parra, A. (2002). Experiencias psi-conflictivas: Una encuesta con implicaciones en parapsicología clínica. Revista Argentina de Psicología Paranormal, 13, 7-47.

Guimaraes Andrade, H. (1988). Poltergeist: Algunas de suas ocurrencias no Brasil. Sao Paulo: Pensamento.

Houran, J. \& Lange, R. (Eds.) (2001). Hauntirlgs and Poltergeist: Multidisciplinary perspectives. Jefferson, NC: McFarland.

Irwin, H. J. (2004). An introduction to parapsychology. Jefferson, NC: McFarland.

Jordán Peña, J. L. (1982). Casas encantadas: Poltergeist. Barcelona: Noguer.

Machado, F. R. \& Zangari, W. (1995). Conversando sobre casas mal-assombradas: O fenomeno poltergeist. Sao Paulo: Edicoes Paulinas.

Nicholls, A. \& Rool, W. G. (2000). Psychological and electromagnetic aspects of haunts. Procedings of presented papers. The Parapsychological Association 43th Annual Convention, 364-378.

Nichols, A. \& Roll, W. G. (1999). A haunting at an army post. Procedings of present papers. The Parapsychological Association 43th Annual Convention, 253-270.

Owen, A. R. G. (1964). Can we explain the poltergeist? Nueva York: Garrett Publications.

Parra, A. (2004). PK occurrences, epilepsy and repressed aggression: analysis of 
Andrés Venier case. Paranormal Review, 32, 23-27.

Percia de Carvalho, A. (1992). Las casas embrujadas: Poltergeist. Valencia: Tetragrama.

Rogo, D. S. (1979). The poltergeist experiences: Investigations into ghostly phenomena. Nueva York: Penguin Books.

Rogo, D. S. (1986). On the track of the poltergeist. Englewood Cliffs, NJ: Prentice-Hall.

Roll, W. G. (1964). The psi field. Proceedings of the Parapsychological Association, 1, 32-64.

Roll, W. G. (1972). Poltergeist. Nueva York: New American Library.

Roll, W. G. (1968). Some physical and psychological aspects of a series of poltergeist phenomena. Journal of the American Society for Psychical Research, 62, 263-308.

Roll, W. G. (1970). Poltergeist phenomena and interpersonal relations. Journal of the American Society for Psychical Research, 64, 66-99.

Roll, W. G. (1972). The poltergeist. Nueva York: Nelson Doubleday.

Roll, W. G. (1977). Poltergeists. En: Wolman, B. B. (Ed.). Handbook of parapsychology (382-413). Nueva York: Van Nostrand Reinhold.

Roll, W. G. (1993). The question of RSPK vs. fraud in the case of Tina Resch. En: Roe, C. R. (Ed.). Proceedings of presented papers. The Parapsychological Association 36th Annual Convention, 456-482.
Roll, W. G., Burdick \& Joines, W. T. (1973). Radial and tangential forees in the Miami poltergeist. Journal of the American Society for Psychical Research, 67, 267-281.

Roll, W. G., Burdick \& Joines, W. T. (1974). The rotating beam theory and the Olive HiII poltergeist. En: Roll, W. G.; Morris, R. L. \& Morris, J. (Eds.). Research in parapsychology 1973 (6467). Metuchen, NJ: Scarecrow.

Roll, W. G. \& Gearhart, L. (1974). Geomagnetic perturbations and RSPK. En: Roll, W. G.; Morris, R. L. \& Morris, J. (Eds.). Research in parapsychology 1973 (44-46). Metuchen, NJ: Scarecrow.

Roll, W. G.; Maher, M. \& Brown, B. (1992). An investtigation of reported haunting occurrences in a japanese restaurant in Georgia. En: Williams Cook, W. (Ed.). Research in Parapsychology 1992 (56-62). Lanham, MD: The Scarecrow Press.

Roll, W. G. \& Montagno, E. (1983). Similarities between RSPK and psychomotor epilepsy. En: Roll, W. G.; Beloff, J. \& White, R. A. (Eds.). Research in parapsychology 1982 (270-271). Metuchen, NJ: Scarecrow.

Roll, W. G. \& Persinger, M. (1998). Poltergeist and nonlocality: Energetic aspects of RSPK. En: Roe, C. R. (Ed.). Proceedings of the 41th Annual Convention of the Parapsychological Association (184-199). Halifax, Nova Scotia, Canadá: The Parasychological Association.

Roll, W. G. \& Pratt, J. G. (1971). The Miami disturbances. Journal of the American Society for Psychical Research, 65, 409-454. 
Roll, W. G., Sheehan, L. C., Persinger, M. A., \& Glass, A. Y. (1996). The haunting of White Ranch. The Parapsychological Association 39th Annual Convention, 294.

Roll, W. G. \& Stump, J. (1969). The Olive Hill poltergeist. Proceedings of the $\mathrm{Pa}$ rapsychological Association, 6, 57-58.

Roll, W. G. \& Tringale, S. (1983). A haunting-type RSPK case in New England. En: Roll, W. G.; Beloff, J. \& White, R. A. (Eds.). Research in parapsychology 1982 (132-136). Metuchen, NJ: Scarecrow.

Spencer, J. \& Spencer, A. (1997). The poltergeist phenomenon: An investigation into psychic disturbance. Londres: Headline Book.

Stander, P. \& Schmolling, P. (1996). Poltergeist \& the paranormal: Fact beyond fiction. St. Paul, MN: Lleweling Publications.
Thurston, H. (1953). Ghost and poltergeist. Londres: Burns Oates.

Tinoco, C. A. (1978). Fenómenos de psicociencia espontánea. Manaus: Edición del autor.

Tizané, E. (1977). Le mystere des maisons hanteés. París: Tchou.

Williams, B. J. (2001). The ghost in the mind: A brainenviroment interaction model of the apparitional experience. En: Alvarado, C. S. (Ed.). Proceedings of the Parapsychological Association 44th Annual Convention (364-376). Nueva York.

Wiseman, R.; Watt, C.; Greenling, E.; Stevens, P. \& O'Keefe, C. (1997). An investigation into the alleged haunting of Hampton Court Palace: Psychogical variables and magnetic fields. En: Alvarado, C. S. (Ed.). Proceedings of the Parapsychological Association 44th Annual Convention. (393-407). Nueva York. 